This document is the Accepted Manuscript version of a Published Work that appeared in final form in Langmuir, copyright $\odot$ American Chemical Society after peer review and technical editing by the publisher. To access the final edited and published work see https://doi.org/10.1021/la3037296 


\section{Channel Blocking of MspA Revisited}

Ayomi S. Perera*1, Hongwang Wang ${ }^{1}$, Matthew T. Basel ${ }^{2}$, Megh Raj Pokhrel $^{3}$, Pubudu Siyambalagoda Gamage ${ }^{1}$, Mausam Kalita ${ }^{1,2}$, Sebastian Wendel ${ }^{1}$, Bryan Sears ${ }^{4}$, Dhanushi

Welideniya ${ }^{1,}$ Yao Liü, Christer Aakeröy ${ }^{1}$, Claudia Turro ${ }^{* 4}$, Deryl L. Troyer ${ }^{2}$ and Stefan H. Bossmann $^{* 1}$

1: Kansas State University, Department of Chemistry, CBC Building 213, Manhattan, KS 66506, sbossman@ksu.edu; 2: Kansas State University, Department of Anatomy\&Physiology, Coles 130, Manhattan, KS 66506; 3: Department of Chemistry, Tribhuvan University, Kathamandu, Nepal; 4: Department of Chemistry and Biochemistry, The Ohio State University, Columbus, $\mathrm{OH} 43210$

KEYWORDS Supramolecular, MspA, Mycobacterium tuberculosis, Mycobacterium smegmatis, channel blocking, zeta potential, AFM, steady state and nanosecond laser-induced absorption and emission spectroscopy 


\section{ABSTRACT}

Porin A from Mycobacterium smegmatis (MspA) is a highly stable, octameric channel protein, which acts as the main transporter of electrolytes across the cell membrane. MspA features a narrow, negatively charged constriction zone, allowing stable binding of various analytes thereby blocking the channel. Investigation of channel blocking of mycobacterial porins is of significance in developing alternate treatment methods for tuberculosis. The concept that Ruthenium(II)quaterpyridinium complexes have the capability to act as efficient channel blockers for MspA and related porins, emerged after very high binding constants were measured by HPLC and steady-state luminescence studies. Consequently, the interactions between the Ruthenium(II) complex RuC2 molecules and MspA, leading to RuC2@MspA assemblies, have been studied utilizing time resolved absorption/emission, AFM, Dynamic Light Scattering (DLS), zeta potential measurements, and Isothermal Titration Calorimetry. The results obtained provide evidence for the formation of clusters/large aggregates of RuC2 and MspA. The results are of interest with respect to utilizing prospective channel blockers in porins. The combination of results from conceptually different techniques shed some light onto the chemical nature of MspA-channel blocker interactions thus contributing to the development of a paradigm for channel blocking. 


\section{INTRODUCTION}

Tuberculosis (TB) is, according to the World Health Organization (WHO), the second most dangerous infectious disease in the world. ${ }^{1}$ Approximately one third of the world's population is infected with M. tuberculosis and 10\% of the infected human subjects will develop active TB during their lifetime. In 2010, TB incidence and prevalence were estimated at 8.8 and 12 million cases respectively. An astonishing number of 1.1 million HIV-negative and 0.35 million HIVpositive humans have succumbed to the disease in the same year. ${ }^{1}$ During the last three decades, multi-resistant strains have appeared due to the decision of many nations to discontinue the treatment of tuberculosis, a practice that threatens all countries that experience immigration. ${ }^{1}$ New hope for tuberculosis patients arises from recent massive drug discovery efforts to develop new TB drugs using either target-based or phenotypic screens. ${ }^{2}$ Gatifloxacin, moxifloxacin, metronidazole or linezolid, which are already in use against other bacterial infections, are currently being evaluated for the treatment of TB in phase 2 or 3 clinical trials. Furthermore, there are at least an additional ten compounds in clinical trials and novel strategies for the development of new molecules are being discussed. ${ }^{3}$ One of these concepts, which will be revisited here is the blocking of mycobacterial channels. Mycobacteria possess an especially thick outer membrane (called "cell envelope"), which acts as a hydrophobic shield against antibiotics. ${ }^{4}$ Channel proteins ("porins") form the main hydrophilic pathways through the cell envelope. ${ }^{4}$ The homo-octameric porin MspA (porin A from Mycobacterium smegmatis) enhances the permeability of cell envelopes for hydrophilic solutes. ${ }^{6}$ The porin MspA features a double ring of eight aspartates (D90 and D91) in its constriction zone (see Figure 1). ${ }^{1}$ This site in MspA appeared to be most attractive for the binding of various cationic substrates, such as cationic resorcin-arenes, ${ }^{2}$ Ruthenium(II)polypyridyl complexes ${ }^{3,4}$ and gold nanoparticles. ${ }^{5,6}$ 
Channel blocking of mycobacteria leads is, principally, able to induce dormancy, because their pathway(s) for taking in hydrophilic nutrients are blocked. Furthermore, the enhanced expression of porins is able to destabilize the mycobacterial cell wall, which increases their susceptibility to antibiotics. This has been shown for M. bovis and M. tuberculosis. ${ }^{12}$ It should be noted that MspA is one of the most stable porins to date; as such it represents an excellent building block for future applications in bioelectronics ${ }^{11}$ and biophotonics. ${ }^{10}$ The formation of supramolecular adducts between Ruthenium(II)quaterpyridinium complexes and MspA was observed, demonstrating that MspA is capable of acting as host for fluorescent guests. ${ }^{9}$ The resulting luminescent adducts have potential applications as fluorescent building blocks for advanced nanoarchitectures. ${ }^{9-11}$ Here, we would like to revisit these experiments and compare them with recent investigations by AFM, time-resolved (ns) absorption, emission spectroscopy, dynamic light scattering, and isothermal titration calorimetry. The purpose of this study is to combine the results from conceptually different techniques towards a comprehensive paradigm of MspAchannel blocker interactions. The lessons from our studies apply virtually to all supramolecular systems that are made from proteins and fluorescent nanostructures. 

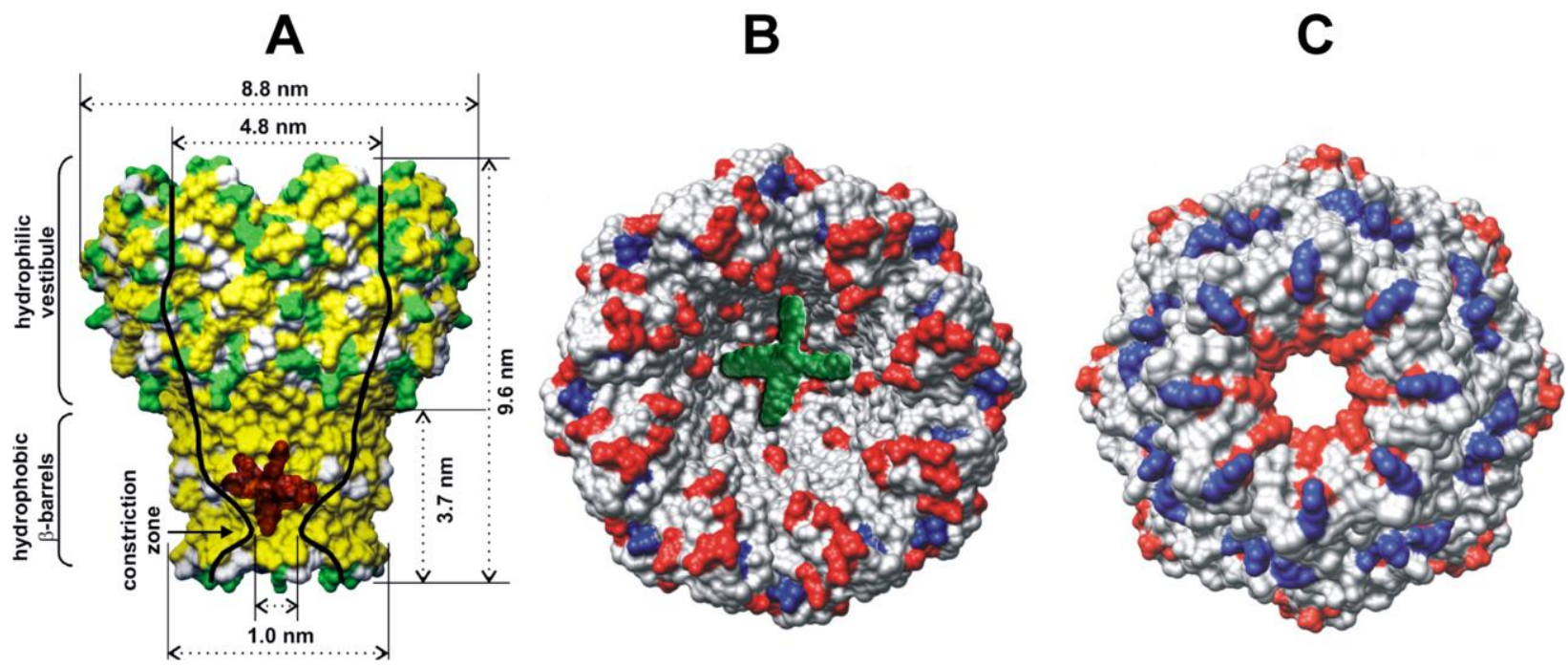

Figure 1: Structure of the homo-octameric mycobacterial porin MspA and the Ruthenium(II)quaterpyridinium complex RuC2 (Ruthenium(2+)-tris-(1,1"'-bis(2-carboxyethyl)$\left[4,4^{\prime}: 2^{\prime}, 2^{\prime \prime}: 4^{\prime \prime}, 4^{\prime \prime \prime}\right.$-quaterpyridine- $\left.\kappa N^{1^{1}}, \kappa N^{1 "}\right]-1,1^{\prime \prime \prime}$-diium). A) MspA is $9.6 \mathrm{~nm}$ in length and $8.8 \mathrm{~nm}$ in width. It's "docking zone", which is formed by hydrophobic $\square$ eta-barrels, is located at the "stem". ${ }^{6}$ The channel blocker RuC2 is shown in red at the constriction zone. B) Structural model of the MspA pore viewed from the top generated using the UCSF Chimera software. The channel blocker RuC2 is shown in green. Negatively and positively charged amino acids are shown in red and blue, respectively. Other amino acids are shown in gray. C) MspA pore viewed from the bottom.

\section{RESULTS AND DISCUSSION}

\section{Photophysical Measurements}

MspA can serve as host system for the four highly-charged Ruthenium(II)-polypyridyl complexes RuC1-RuC4 shown in Figure 2, because it possesses 16 aspartates in its constrictionzone of approx. $1 \mathrm{~nm}$ in diameter. ${ }^{6,8} \mathrm{RuC1}-\mathrm{RuC} 4$ are bound at different locations within MspA, because the interior of MspA is funnel shaped and the complexes range in diameter from 2.43 to $3.18 \mathrm{~nm}$, as determined from the program Chemdraw 3D using a refined set of parameters. ${ }^{9}$ RuC2, RuC3 and RuC4 show significant increase in luminescence and shift in the absorption maxima in the presence of MspA, indicative of binding of the Ruthenium(II)quaterpyridinium complexes to MspA. ${ }^{9}$ Measurements of the corresponding supramolecular binding constants by 
size exclusion chromatography resulted in very high values that range from $1.1 \times 10^{9} \mathrm{M}^{-1}(\mathrm{RuC} 4)$ to $7.5 \times 10^{9} \mathrm{M}^{-1}(\mathrm{RuC} 1)$. These binding constants were calculated assuming a 1:1 stoichiometry of the MspA and the corresponding Ru(II)-complex. ${ }^{9}$ However, recent results obtained from AFM, Dynamic Light Scattering, and Isothermal Titration Calorimetry are not in agreement with a 1:1 stoichiometry, as will be discussed below.

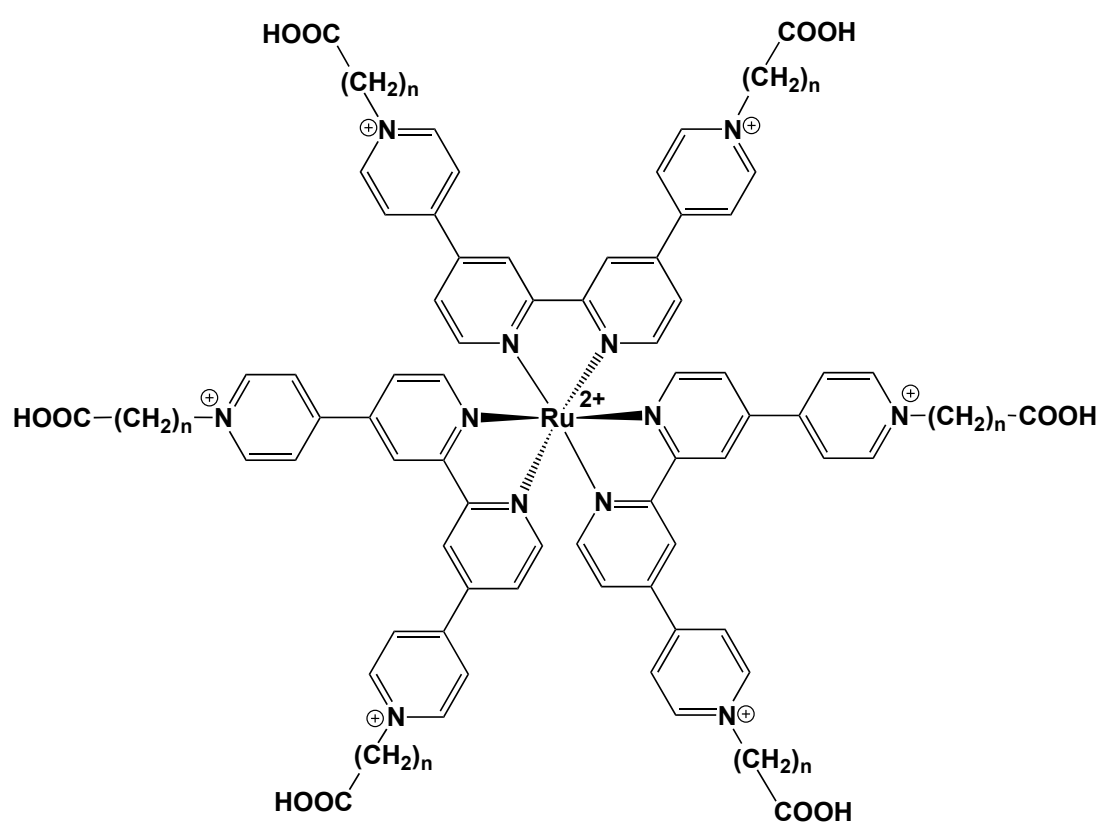

Figure 2: Structures of the Ruthenium(II)quaterpyridinium complexes $\mathrm{RuC1}\left(\left(-\mathrm{CH}_{2}\right)_{1-}\right.$, complex diameter $=2.43 \mathrm{~nm}), \mathrm{RuC} 2\left(\left(-\mathrm{CH}_{2}\right)_{2}-\right.$, complex diameter $\left.=2.92 \mathrm{~nm}\right), \mathrm{RuC} 3\left(\left(-\mathrm{CH}_{2}\right)_{3}-\right.$, complex diameter $=2.99 \mathrm{~nm})$, and $\mathrm{RuC} 4\left(\left(-\mathrm{CH}_{2}\right)_{4-}\right.$, complex diameter $\left.=3.18 \mathrm{~nm}\right){ }^{8}$ 
Table 1. Steady-state absorption $\left(\lambda_{A B S}\right)$ and emission maxima $\left(\lambda_{E M}\right)$ for $2 \times 10^{-5} \mathrm{M}$ of $\mathrm{RuC1}-$ $\mathrm{RuC} 4$ in PBS alone and their supramolecular adducts in the presence of MspA $\left(10 \mu \mathrm{g} \mathrm{mL}^{-1}\right)$ and relative emission intensity.

\begin{tabular}{ccccccc}
\hline & \multicolumn{2}{c}{$\mathrm{RuC}_{\mathrm{n}}$} & & \multicolumn{2}{c}{$\mathrm{RuC}_{\mathrm{n}} @ \mathrm{MspA}$} & \\
\cline { 2 - 3 } $\mathrm{Ru}(\mathrm{II})$ complex & $\lambda_{A B S}(\mathrm{~nm})$ & $\lambda_{E M}(\mathrm{~nm})$ & & $\lambda_{A B S}(\mathrm{~nm})$ & $\lambda_{E M}(\mathrm{~nm})$ & $I_{R E l^{a}}{ }^{a}$ \\
\hline $\mathrm{RuC1}$ & 491 & 662 & & 663 & 0.95 \\
$\mathrm{RuC2}$ & 490 & 668 & & 673 & 7.25 \\
$\mathrm{RuC3}$ & 479 & 632 & & 643 & 2.55 \\
$\mathrm{RuC} 4$ & 481 & 635 & & 670 & 2.70 \\
\hline
\end{tabular}

${ }^{a}$ Relative emission intensity of MspA-bound and free $\mathrm{RuC}_{\mathrm{n}}$ complex.

The signature steady state absorption and emission maxima of RuC1-C4 in the presence and absence of MspA are summarized in Table 1, along with the relative luminescence intensity of each complex upon binding to MspA. Binding of the Ruthenium(II)quaterpyridinium complexes to MspA leads to bathochromic shifts in the emission, with $11 \mathrm{~nm}$ for RuC3 and $35 \mathrm{~nm}$ for RuC4). The highly charged protein environment surrounding the complex gives rise to the redshift in the absorption and emission; similar effects have been observed in other encapsulated Ruthenium complexes. ${ }^{13}$ In addition, with the exception of $\mathrm{RuC1}$, an increase in the luminescence intensity from the $\mathrm{Ru}(\mathrm{II})$ complexes increases by up to a factor of 7.25 , measured for RuC2, was observed when bound to MspA. Since the sample is in aqueous medium, the luminescence that arises due to the metal to ligand charge transfer of the Ruthenium(II) complex is partially non-radiatively deactivated by the surrounding the water molecules. ${ }^{14}$ However, when the $\mathrm{Ru}(\mathrm{II})$ complexes bind to MspA, the protein environment shields the complex from this deactivation pathway, which leads to an enhancement in the observed luminescence intensity. It is noteworthy that the lifetime of luminescence $\tau_{\mathrm{EM}}$ decreases when the $\mathrm{Ru}(\mathrm{II})$ complexes are bound to MspA (Table 2). These differences between the trends of the emission intensity and 
lifetime of bound complex will be discussed in more detail in later section. However, it may be pointed out here that these findings can be explained by clustering of several $R u(I I)$ complexes when bound to MspA. ${ }^{15}$

The second important finding from time-resolved absorption measurements indicates that MspA is able to participate in electron transfer reactions. The lifetimes of the charge-separated state $\mathrm{Ru}^{\mathrm{III}}-\mathrm{py}^{0}$ of $\mathrm{RuC1}-4$ range from $156 \mathrm{~ns}(\mathrm{RuC1})$ to $42 \mathrm{~ns}(\mathrm{RuC} 4)$. In the presence of MspA, they are significantly reduced, as indicated in Table 2 . Interestingly, $\mathrm{RuC} 1$, which possesses the longest living charge-separated state, shows the shortest lifetime of $\mathrm{Ru}^{\mathrm{III}}-\mathrm{py}^{0}$ in the presence of MspA.

Table 2 Summary of the lifetimes of time-resolved absorption and emission processes of Ruthenium(II)quaterpyridinium complexes in the absence and presence of MspA.

\begin{tabular}{|c|c|c|c|c|c|}
\hline $\mathrm{Ru}(\mathrm{II})$ complex & $\begin{array}{c}\tau_{\mathrm{ET}}(\mathrm{ns}) \\
\mathrm{RuC}\end{array}$ & $\begin{array}{c}\tau_{\mathrm{EM}}(\mathrm{ns}) \\
\mathrm{RuC}_{\mathrm{n}}\end{array}$ & $\begin{array}{c}\tau_{\mathrm{ET}}(\mathrm{ns}) \\
\mathrm{RuC} @ \mathrm{MspA}\end{array}$ & $\begin{array}{c}\tau_{\mathrm{EM}}(\mathrm{ns}) \\
\mathrm{RuC} @ \mathrm{MspA}\end{array}$ & $\begin{array}{c}\mathrm{R}_{\mathrm{n}} @ \square \square^{3+2+} \\
\mathrm{V}(\mathrm{vs} . \mathrm{SHE})\end{array}$ \\
\hline $\mathrm{RuC} 1$ & 156 & 753 & 8.2 & 423 & 1.51 \\
\hline $\mathrm{RuC} 2$ & 110 & 770 & 18.2 & 405 & 1.57 \\
\hline $\mathrm{RuC} 3$ & 64 & 862 & 20.5 & 388 & 1.55 \\
\hline $\mathrm{RuC} 4$ & 42 & 816 & 22.4 & 392 & 1.54 \\
\hline
\end{tabular}

$\tau_{\mathrm{ET}}\left(\mathrm{RuC}_{\mathrm{n}}\right)$ : lifetime of the charge separated state $\mathrm{Ru}^{\mathrm{IIII}}-\mathrm{py}^{0}$ of $\mathrm{RuC}_{\mathrm{n}}\left(2 \times 10^{-5} \mathrm{M}\right.$ in PBS$)$. The transient absorption was recorded at $\lambda=590 \mathrm{~nm}$.

$\tau_{\mathrm{ET}}\left(\mathrm{RuC}_{\mathrm{n}} @ \mathrm{MspA}\right)$ : lifetime of the charge separated state $\mathrm{Ru}^{\mathrm{III}}-\mathrm{py}^{0}$ of $\mathrm{RuC}_{\mathrm{n}}\left(2 \times 10^{-5} \mathrm{M}\right.$ in PBS $)$ in the presence of MspA $\left(10 \mu \mathrm{g} \mathrm{mL}^{-1}\right)$. The transient absorption was recorded at $\lambda=590 \mathrm{~nm}$.

$\tau_{\mathrm{EM}}\left(\mathrm{RuC}_{\mathrm{n}}\right)$ : lifetime of the ${ }^{3} \mathrm{MLCT}$ emission of $\mathrm{RuC}_{\mathrm{n}}\left(2 \times 10^{-5} \mathrm{M}\right)$ in aerated PBS $\tau_{\mathrm{EM}}\left(\mathrm{RuC}_{\mathrm{n}} @ \mathrm{MspA}\right)$ : lifetime of the ${ }^{3} \mathrm{MLCT}$ emission of $\mathrm{RuC}_{\mathrm{n}}\left(2 \times 10^{-5} \mathrm{M}\right)$ in aerated PBS in the presence of MspA $\left(10 \mu \mathrm{g} \mathrm{mL}^{-1}\right)$.

The redox potentials have been determined by differential pulse voltammetry and are given vs. the standard hydrogen electrode (SHE) 
The plot of the relative luminescence increase of RuC1-RuC4 when bound to MspA IREL vs. the relative decrease in lifetime of the corresponding complex when bound to MspA $\tau_{R E L}\left[\tau_{R E L}=\right.$ $\left.\tau\left(\mathrm{RuC}_{\mathrm{n}} @ \mathrm{MspA}\right) / \tau\left(\mathrm{RuC}_{\mathrm{n}}\right)\right]$ indicates that there is no straightforward correlation between the steady-state and time-resolved luminescence from $\mathrm{RuC} 1-\mathrm{RuC} 4$ in the presence and absence of MspA. We have interpreted this result as indication of possible clustering of more than one $\mathrm{Ru}(\mathrm{II})$ complex either within the inner pore or at the surface of MspA.

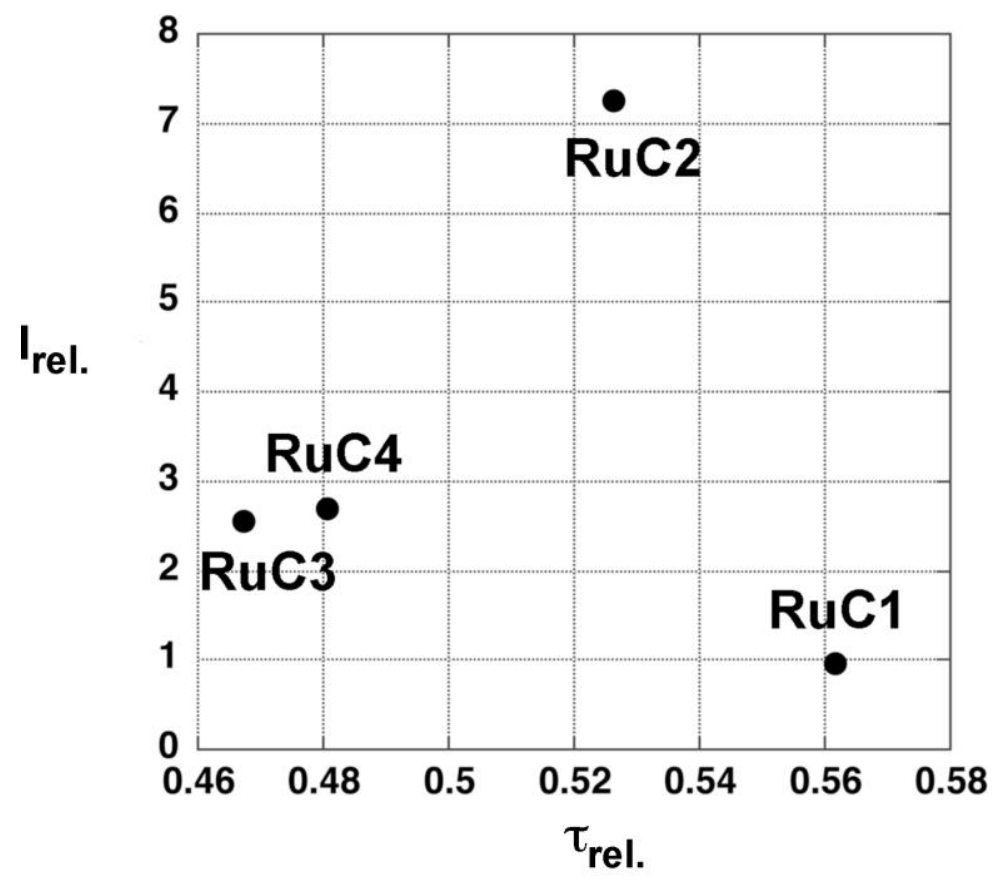

Figure 3: Plot of the steady-state luminescence enhancement of the $\mathrm{Ru}(\mathrm{II})$ quaterpyridinium complexes RuC1-4 (Irel., Table 1) vs. the ratio of their emission lifetimes in the presence and absence of MspA ( $\left.\left(\mathrm{RuC}_{\mathrm{n}} @ \mathrm{MspA}\right) / \tau\left(\mathrm{RuC}_{\mathrm{n}}\right)\right)$.

Scheme 1 summarizes the photophysical processes that have been observed in $\mathrm{RuC}_{\mathrm{n}} @ \mathrm{MspA}$ adducts: absorption of light (h $\left.v_{\mathrm{ABS}}\right)$ by each of the four Ruthenium(II)quaterpyridinium complexes $\left(\mathrm{Ru}^{\mathrm{II}}-\right.$ py $\left.{ }^{+}\right)$results in the population of their respective ${ }^{3}$ MLCT-states $\left({ }^{*} \mathrm{Ru}^{\mathrm{II}}-\mathrm{py}^{+}\right)$. From there, internal conversion (not shown), luminescence to the ground state $\left(\mathrm{Ru}^{\mathrm{II}}-\mathrm{py}^{+}\right)$and electron transfer to the charge-separated state $\mathrm{Ru}^{\mathrm{III}}-\mathrm{py}^{0}\left({ }^{3} \mathrm{CS}\right)$ were observed. In the presence of 
MspA, the lifetime of the charge-separated state is significantly shortened, indicating that MspA can participate in electron transfer reactions. The tyrosine-units in MspA $\left(\mathrm{E}^{0}=+0.93 \mathrm{~V} v s\right.$ NHE at $\mathrm{pH}=7)^{16}$ have the capability to act as electron donors to reduce $\mathrm{Ru}^{\mathrm{III}}$ to $\mathrm{Ru}^{\mathrm{II}}\left(\mathrm{E}^{0}=+1.57 \mathrm{~V}\right.$ $(\mathrm{RuC} 2)$ to $+1.51 \mathrm{~V}(\mathrm{RuC} 1)$ vs $\mathrm{NHE})$ present in the ${ }^{3} \mathrm{MLCT}$ or ${ }^{3} \mathrm{CS}$ states. The MspA-octamer contains 32 tyrosines residues at positions $48,66,82$, and $177 .{ }^{6}$ In addition, the clustering of $\mathrm{RuC1}$-RuC4 within the MspA environment can lead to annihilation of the charge-separated states to produce ${ }^{3} \mathrm{MLCT}$ (metal to ligand charge transfer) and ${ }^{1} \mathrm{GS}$ (ground state) from each event, resulting in greater ${ }^{3} \mathrm{MLCT}$ emission in the presence of MspA while retaining a shorter emission lifetime. This annihilation process also explains the shorter lifetime of the CS state in MspA as compared to free complex. It should be noted that owing to the different sizes of RuC1-RuC4, this clustering likely differs from one complex to another. Moreover, evidence from AFM and dynamic light scattering discussed in later sections also indicates that clustering is not only found within the pore of MspA, but also on its outer surface.

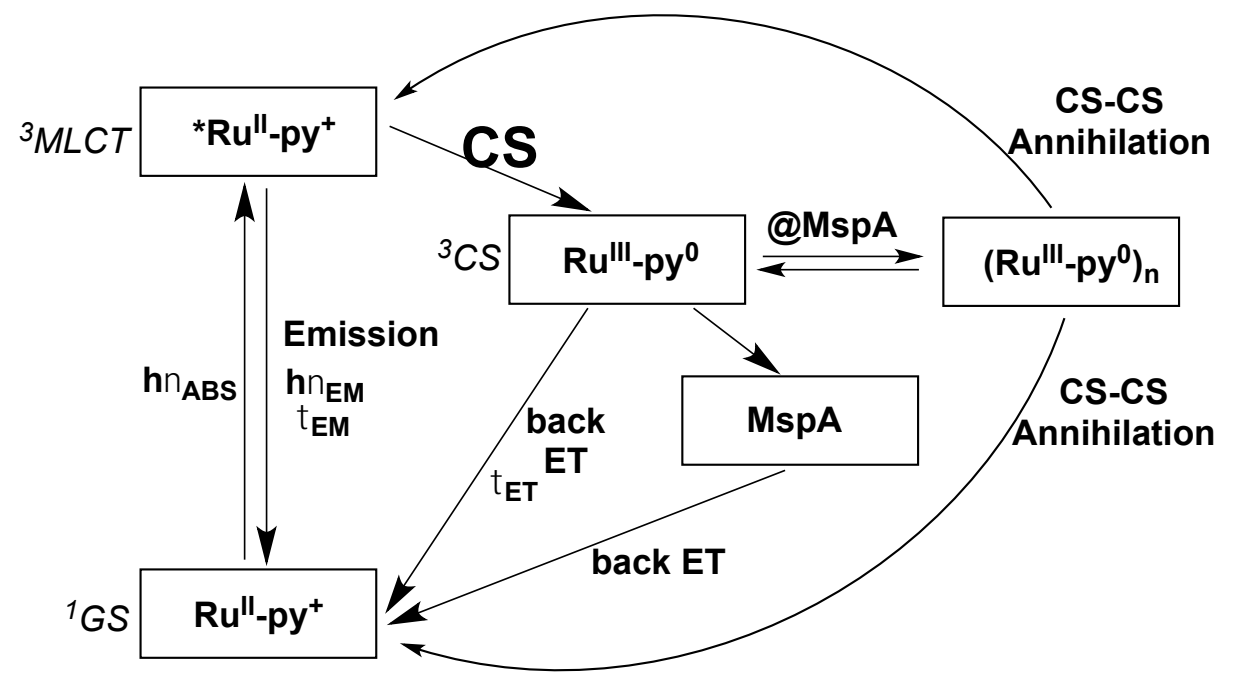

Scheme 1. Proposed photophysical processes for RuC1@MspA - RuC4@MspA. 


\section{Aggregation of MspA and RuC2 by Atomic Force Microscopy}

Intrigued by the inconsistencies in the steady-state and time-resolved luminescence experiments for RuC1-RuC4 in the absence and when bound to MspA, which suggest that the stoichiometry of $\mathrm{Ru}(\mathrm{II})$ quaterpyridinium complexes and MspA is not a simple 1:1, we performed Atomic Force Microscopy (AFM) studies of RuC2 and MspA on mica. AFM images were recorded in the magnetic ac mode (MACmode) ${ }^{17}$ and measurements were performed at the micaair interface. AFM is the method of choice to determine the topography of a sample. Furthermore, there exists a discernible sensitivity of the phase of the cantilever oscillations to the tip-sample interaction forces, such that changes in the phase of the oscillations can be used to discriminate between materials possessing different viscoelastic properties. Figure 4a shows that single MspA pores can be imaged on mica using AFM. The large pore opening of MspA is directed outward, whereas the loop region and the constriction zone are directed toward the mica support. When in the MACMode (oscillation frequency $75 \mathrm{kHz}$, air), the oscillating AFM probe conveys a force of approximately $25 \mathrm{pN}$, which does not distort the protein's structure. This result is in agreement with earlier findings. ${ }^{11}$ It is noteworthy that $\sim 90 \%$ of the MspA octamers appear to be standing "upright" on mica when deposited from PBS buffer, compared to $\sim 98 \%$, oriented in the same manner when deposited from 20:80 PBS:methanol (v:v) (PBS = phosphate buffered saline). As molecular modeling indicates (see Figure 1c), the "bottom" of MspA, including the periplasmatic loop region, bears a significantly higher density of positive charges than the upper vestibule or the interior of MspA. Therefore, electrostatic attraction between MspA and mica $\left(\xi=-87 \mathrm{mV} \text { at } \mathrm{I}=1 \times 10^{-3} \mathrm{M} \text { and }-38 \mathrm{mV} \text { at } \mathrm{I}=1 \times 10^{-2} \mathrm{M} \text { at } \mathrm{pH}=7.4\right)^{18}$ is most likely responsible for the observed orientation of MspA. Figure $4 \mathrm{~b}$ shows the AFM image of the supramolecular adduct between MspA and $\mathrm{RuC} 2$. In the absence of a host, residual water remains within the upper vestibule of MspA even if AFM measurements are performed at the 
mica-air interface where MspA is adsorbed. The presence of water leads to a discernible shift in the phase of the cantilever oscillations due to the tip-sample interaction forces, which renders the pores detectable. It is clearly discernible in Figure $4 \mathrm{~b}$ that the pore of MspA can no longer be detected due to partial blocking of the MspA pore by RuC2, most likely because of changes in the biomechanical properties due to binding of a $\mathrm{Ru}(\mathrm{II})$ complex featuring up to 8 positive charges. It is our hypothesis that this process is leading to an electrostatic contraction of the pore and potentially extrusion of some of the bound water.

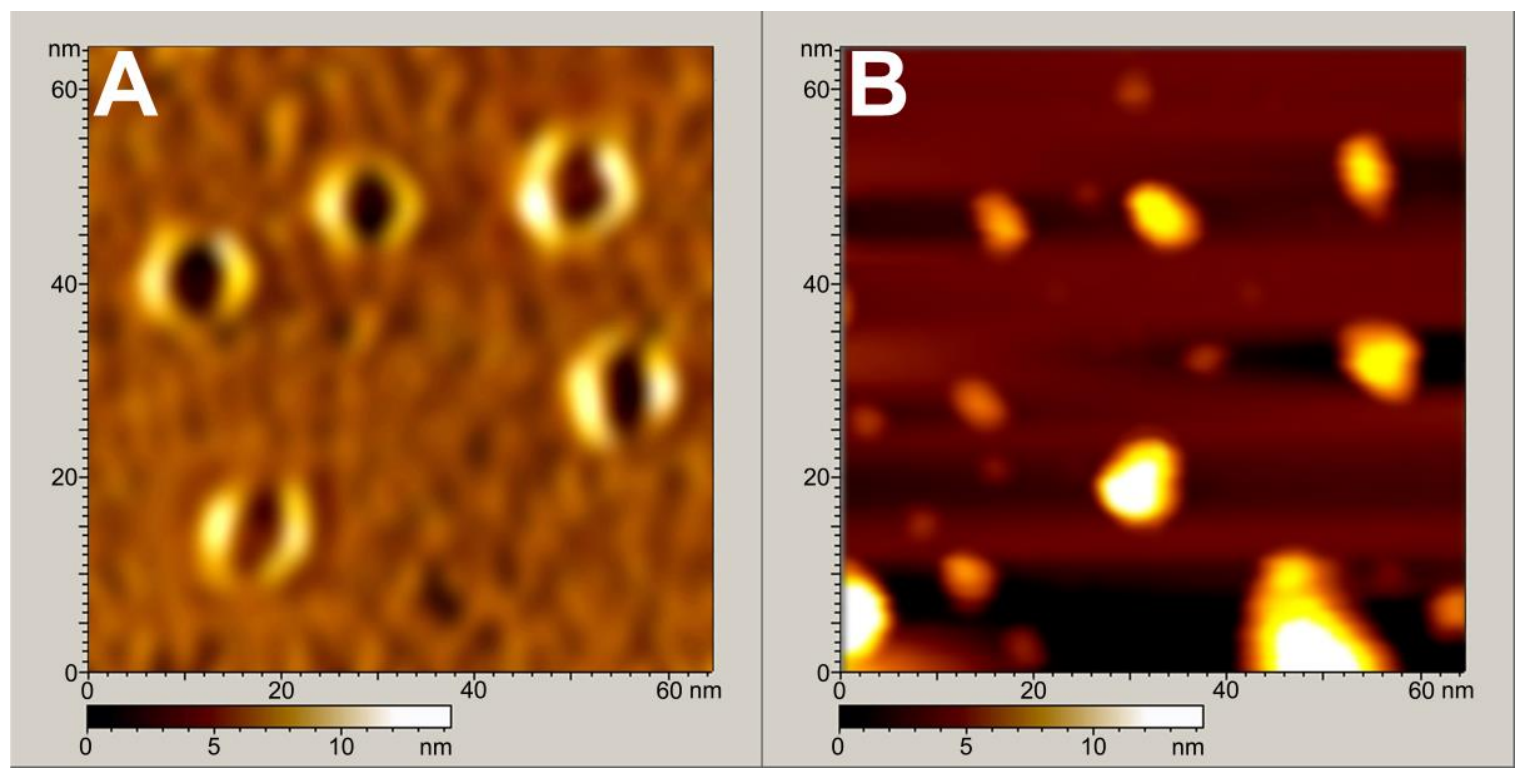

Figure 4: A: MspA on Mica after deposition (spin-casting) from 20:80 PBS:methanol (v:v). B: RuC2 and RuC2@MspA aggregates. Note the open MspA-pores in the absence of RuC2 in 4A. In Figure 4B, all MspA-pores are closed due to RuC2-binding within MspA. Note that RuC2 (smaller structures) and RuC2@MspA aggregates can be discerned. Further explanations are provided in the text.

In the presence of MspA all nanostructures analyzed had heights of $10 \pm 2 \mathrm{~nm}$ and the height of unbound $\mathrm{RuC} 2$ was determined to $3.0 \pm 0.5 \mathrm{~nm}$. Although the height measurements by AFM are very precise, the measurement of width less exact, because the AFM tip has a diameter of $\sim 7$ $\mathrm{nm}$, which is comparable to the width of MspA $(\mathrm{d}=8.8 \mathrm{~nm})$. Since RuC2 is "only" $2.92 \mathrm{~nm}$ in 
diameter, it is virtually impossible to determine how many $\mathrm{RuC} 2$ complexes are bound to one MspA protein by observing one or a few supramolecular RuC2@MspA aggregates. Therefore, we performed a series of AFM measurements of RuC2 alone, MspA alone, and mixtures of $\mathrm{RuC2} / \mathrm{MspA}$ at molar ratios of $10 / 1$ and $100 / 1$ on mica. The solutions of RuC2 and/or MspA in 1X PBS were spin-cast and AFM-measurements were conducted after drying the mica plates for $24 \mathrm{~h}$ at $50 \%$ relative humidity at $300 \mathrm{~K}$. The AFM data were analyzed using the program IMAGE, generously provided by the National Institutes of Health. ${ }^{19}$ The results are shown in the Supplementary Information section in Figures SXXX and SXXX.

The AFM data clearly disprove the mechanistic assumption of a 1:1 stoichiometry between of RuC2 and MspA on mica. However, the question remains at this point, whether the observations in the 2-dimensional systems are unique due to the presence of the negatively charged mica surface onto which both MspA and RuC2 can adsorb, or whether a similar behavior can be discerned in the 3-dimensional solution phase.

\section{Aggregation of MspA and RuC2 by Dynamic Light Scattering}

We also investigated the aggregation of individual $\mathrm{MspA}^{20}$ and $\mathrm{RuC2} / \mathrm{MspA}$ mixtures in dilute 1x PBS aqueous solutions as a function of temperature by using dynamic light scattering. ${ }^{21}$ In a previous report, we showed that MspA has a distinct tendency to aggregate with increasing temperature, ${ }^{20}$ and the maximum diameter of the MspA vesicles was $\sim 180 \mathrm{~nm}$ at $312 \mathrm{~K}$. We established that MspA aggregation proceeds due to hydrophobic interactions between the docking zones of the proteins (see Figure 1a). Although hydrophobic interaction is the major mechanism behind the aggregation behavior of MspA, we also found evidence of contributions from hydrogen bonding and/or ionic interactions to the supramolecular behavior of MspA. In 
particular, the packing parameter ${ }^{21}$ of $\mathrm{MspA}$ is $\mathrm{P}=0.31$, which is indicative of surfactants forming spherical or ellipsoidal micelles, since the packing parameters of surfactants that form bilayer vesicles are close to $1 .{ }^{22}$ However, MspA forms vesicles and not micelles in PBS. Since the formation of vesicles has been clearly proven by TEM, the observed deviation of predicted and detected supramolecular aggregation behavior of MspA can be explained by assuming interaction of the polar outer surfaces of MspA's vestibules with each other when forming vesicles. ${ }^{22}$

Interestingly, MspA forms much larger clusters in the presence of $\mathrm{RuC2}$, as evidenced by dynamic light scattering (DLS). At $298 \mathrm{~K}$, all three mixtures of MspA and RuC2 have size maxima of $315 \pm 20 \mathrm{~nm}$, and for all three ratios of RuC2 to MspA, a steady decrease of the supramolecular aggregates in size was observed as a function of increasing temperature, and the diameters converge at $190 \pm 15 \mathrm{~nm}$ at $343 \mathrm{~K}$. It is noteworthy that RuC2/MspA=10/1 and 100/1 samples form aggregates of very similar size in the temperature range 300 to $344 \mathrm{~K}$ that are experimentally indistinguishable, whereas the $\mathrm{RuC} 2 / \mathrm{MspA}=1000 / 1$ mixture forms significantly larger aggregates. 


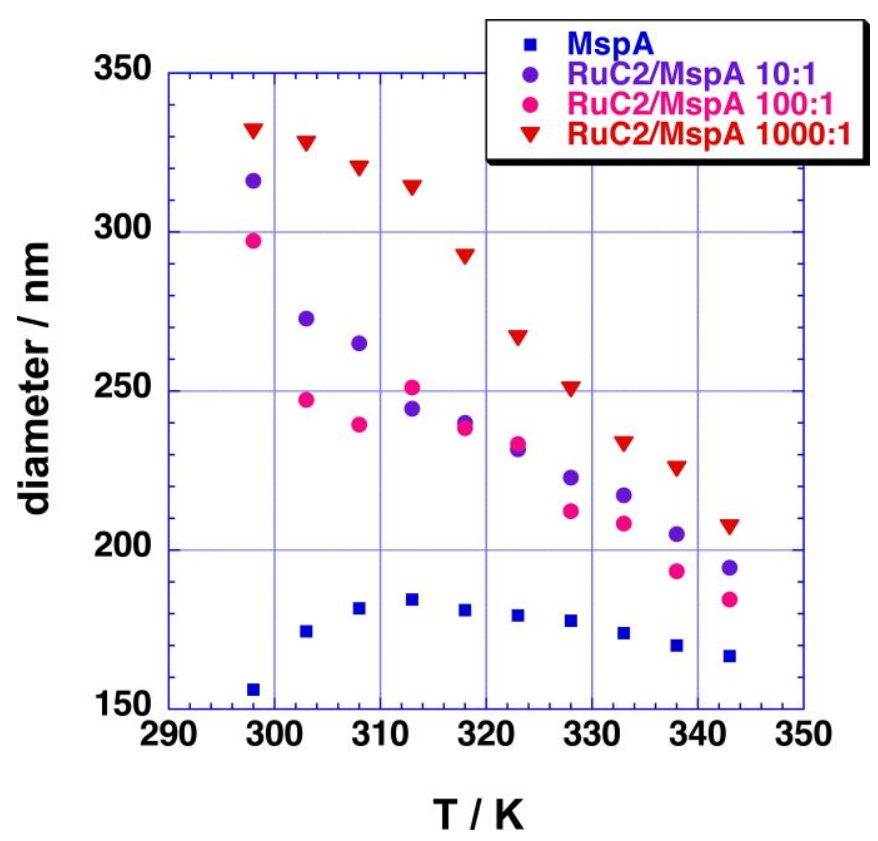

Figure 5. Hydrodynamic diameter of MspA aggregates as a function of temperature measured by dynamic light scattering (error $\pm 8 \mathrm{~nm}$ ): MspA $\left(1.688 \times 10^{-5} \mathrm{mg} \mathrm{mL}^{-1}\right)$ in $1 \mathrm{X}$ PBS and mixtures of $\mathrm{RuC} 2$ and MspA prepared with $[\mathrm{MspA}]=1.875 \times 10^{-10}$ moles and varying concentrations of $\mathrm{RuC} 2$ to attain RuC2/MspA ratios of $10 / 1,100 / 1$ and $1000 / 1$ in $2.0 \mathrm{ml}$ of $1 \mathrm{x}$ PBS. The 10:1 mixture was prepared to duplicate the concentrations used for the channel blocking experiments conducted previously.

The data summarized in Figure 5 indeed provides strong evidence that MspA does not form 1:1 aggregates with $\mathrm{RuC} 2$ and other positively charged $\mathrm{Ru}(\mathrm{II})$ complexes that have been discussed as potential channel blockers. For the 1:1 stoichiometry, the hydrodynamic parameter of the RuC2@MspA aggregates would not be expected to vary because RuC2 is bound in the interior of MspA, as indicated in Figure 1a, would not result in a diameter change. In reality, MspA forms large supramolecular structures of varying stoichiometry with $\mathrm{Ru}(\mathrm{II})$ complexes and not 1:1 aggregates. A fraction of the Ru(II) complexes may be bound in MspA's interior channel, as AFM indicates, but it is clear from the data that this is not the sole mode of binding! As a consequence, $\mathrm{Ru}(\mathrm{II})$ complexes are expected to be less suitable channel blockers for the 
treatment of $\mathrm{TB}$, which casts a serious shadow of doubt on their suitability as water-soluble antimycobacterial drugs.

Zeta-Potential Measurements of MspA and RuC2/MspA Aggregates as a Function of Temperature

The surface charge of the MspA and RuC2/MspA aggregates as a function of temperature was investigated by means of a series of zeta potential measurements by electrophoretic light scattering ${ }^{23}$, and the results are shown in Figure 6 . The zeta potential is defined as the electric potential between the slipping plane in the interfacial double layer and the bulk solution. ${ }^{23}$

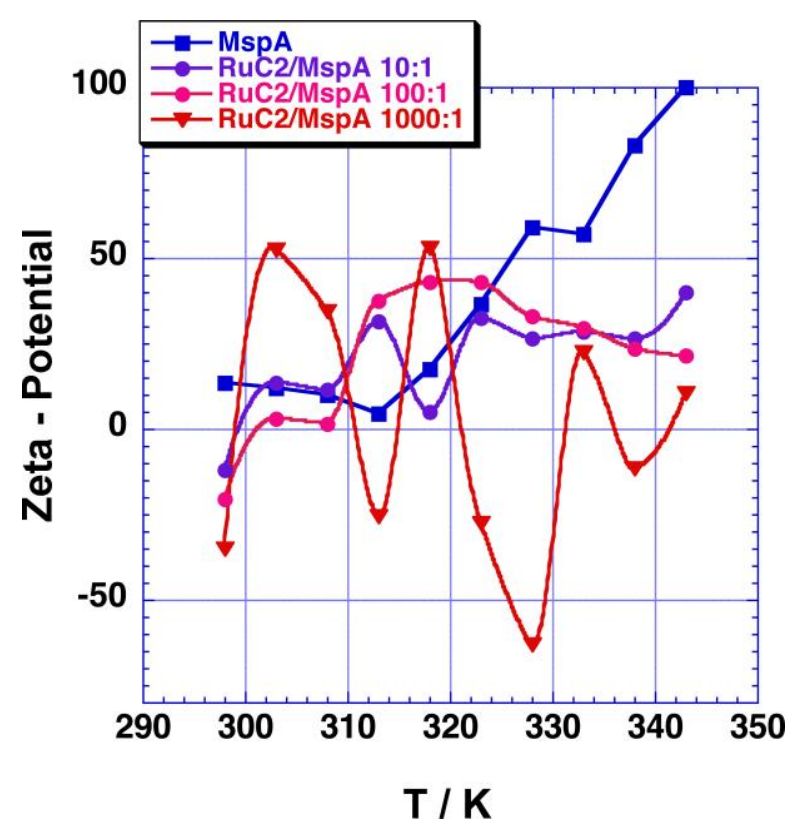

Figure 6. Zeta potential of MspA and $\mathrm{RuC} 2 / \mathrm{MspA}$ aggregates as a function of temperature for MspA (1.688 x $\left.10^{-5} \mathrm{mg} \mathrm{mL}^{-1}\right)$ in 1x PBS and mixtures of RuC2 and MspA prepared with [MspA] $=1.875 \times 10^{-10}$ moles and varying concentrations of RuC2 to attain RuC2/MspA ratios of 10/1, $100 / 1$ and $1000 / 1$ in $2.0 \mathrm{ml}$ of $1 \mathrm{X}$ PBS. 
The zeta potential $\zeta$ of MspA vesicles has been reported and discussed in an earlier report. ${ }^{20} \mathrm{In}$ 1X PBS, the $\zeta$ value of MspA is slightly positive $(\zeta=10 \pm 14 \mathrm{mV})$ in the low temperature range ( 296 to $320 \mathrm{~K}$ ). Above $320 \mathrm{~K}$, a remarkable increase of $\zeta$ to $100 \pm 12 \mathrm{mV}$ at $344 \mathrm{~K}$ is observed. Very high $\zeta$-potentials $(\zeta>40)$ typically indicate excellent ionic stabilization ${ }^{23}$ and the temperature dependence of the $\zeta$ potential is attributed to an endergonic adsorption process of cations $\left(\mathrm{Na}^{+}\right.$and $\left.\mathrm{K}^{+}\right)$at MspA.

Although the dependence of the hydrodynamic diameters on the temperature is strikingly similar for all three mixtures of $\mathrm{RuC} 2$ and MspA investigated, their $\zeta$-potentials as a function of temperature are extremely different. Again the ratios of $\mathrm{RuC} 2 / \mathrm{MspA}=10 / 1$ and 100/1 exhibit similar behavior, and the $\zeta$-potentials for these two samples generally increase as a function of temperature, a feature similar to that observed for MspA alone. It is noteworthy that the $\zeta$ potential of all three $\mathrm{RuC} 2 / \mathrm{MspA}$ mixtures is negative at low temperatures where the largest aggregates are found. The temperature dependence of the $\mathrm{RuC} 2 / \mathrm{MspA}=1000 / 1$ sample is characterized by oscillations of the $\zeta$-potential in the range between +50 and $-70 \mathrm{mV}$. Considering that stable aggregates are formed above $+40 \mathrm{mV}$ and below $-40 \mathrm{mV}$, this experimental finding, which was reproduced three times, is surprising. It is noteworthy that at it is at this mixing ratio the largest RuC2@MspA aggregates are found at $298 \mathrm{~K}$, as measured by DLS (Figure 5). Furthermore, the size of the RuC2@MspA aggregates decreases continuously with increasing temperature. However, the $\zeta$-potentials recorded for the three mixtures do not show a monotonous increase or decrease. The increase of the $\zeta$-potential for MspA alone can be explained with sodium and potassium adsorption from the PBS buffer. The temperature dependence can then be attributed to the reduced size of the RuC2/MspA aggregates at higher 
temperature leading to a greater surface area for cation adsorption. The enhanced macromolecular motion of MspA with increasing temperature may lead to changing dynamics of forming and breaking hydrogen bonds as the temperature increases. The observed decrease in the size of the MspA-vesicles and RuC2@MspA aggregates provides a higher surface for cation adsorption, resulting in the observed remarkable increase of the zeta potential of MspA in $1 \mathrm{X}$ PBS.

RuC2 competes with $\mathrm{Na}^{+}$and $\mathrm{K}^{+}$for the adsorption on the inner and outer surfaces of MspA, as steady-state and time resolved luminescence measurements, time-resolved absorption studies, and AFM measurements have indicated. RuC2 possesses a greater charge than $\mathrm{Na}^{+} / \mathrm{K}^{+}$, which varies between +2 and +8 , depending on the solution $\mathrm{pH}$. At $\mathrm{pH}=7.4$, such as in $\mathrm{PBS}$, we can assume that the complex is fully deprotonated, resulting in an overall charge of +2 , since it features six negatively charged carboxylate groups. The area covered by RuC2 when adsorbed to MspA is $8.6 \mathrm{~nm}^{2}$, corresponding to $\sim 20$ amino acid residues. As a consequence, it can block sites on the surface of MspA, which otherwise would be able to bind $\mathrm{Na}^{+}$or $\mathrm{K}^{+}$. The oscillation of the $\zeta$-potential is most pronounced at the highest concentration of $\mathrm{RuC} 2$. The temperature dependence of the $\zeta$ - potential is indicative of two endergonic adsorption processes, RuC2 vs. $\mathrm{Na}^{+} / \mathrm{K}^{+}$, at MspA. The water-accessible surface of MspA in the observed RuC2@MspA aggregates will be predominantly covered with $\mathrm{RuC} 2$ when the $\zeta$-potential is negative (at temperatures $<298 \mathrm{~K}, 310-315 \mathrm{~K}, 322-334 \mathrm{~K}$, and $337-342 \mathrm{~K}$ ). The positive charges from arginine and protonated lysine residues of MspA, as well as $\mathrm{Na}^{+}$and/or $\mathrm{K}^{+}$adsorption from $\mathrm{PBS}$, cause positive $\zeta$-potentials between 298 and $310 \mathrm{~K}, 315$ and $322 \mathrm{~K}, 334$ and $337 \mathrm{~K}$, and above342 K. Apparently, changes in temperature are able to tip the balance of the competing adsorption of $\mathrm{RuC} 2$ and or $\mathrm{Na}^{+} / \mathrm{K}^{+}$to MspA. In addition to both adsorption processes, the 
deprotonation of the carboxylate side-chains of MspA and the protonation of its amine-side chains are temperature dependent, resulting in the observed (reversible) oscillation of the $\zeta$-potentials as a function of temperature.

Determination of the Binding Constant of RuC2 to MspA by Isothermal Titration Calorimetry

The binding constant of $\mathrm{RuC} 2$ to $\mathrm{MspA}$ has been determined by Isothermal Calorimetric Titration (ITC) to $1.4 \pm 0.1 \times 10^{6} \mathrm{M}^{-1}$ in $1 \times \mathrm{PBS}$ at $298 \mathrm{~K}$. The ratio of RuC2 to MspA that generated the best fit of the experimental data was 9.8 to 1 . We regard this as further proof for the existence of multiple binding sites for $\mathrm{RuC} 2$ at/in MspA.

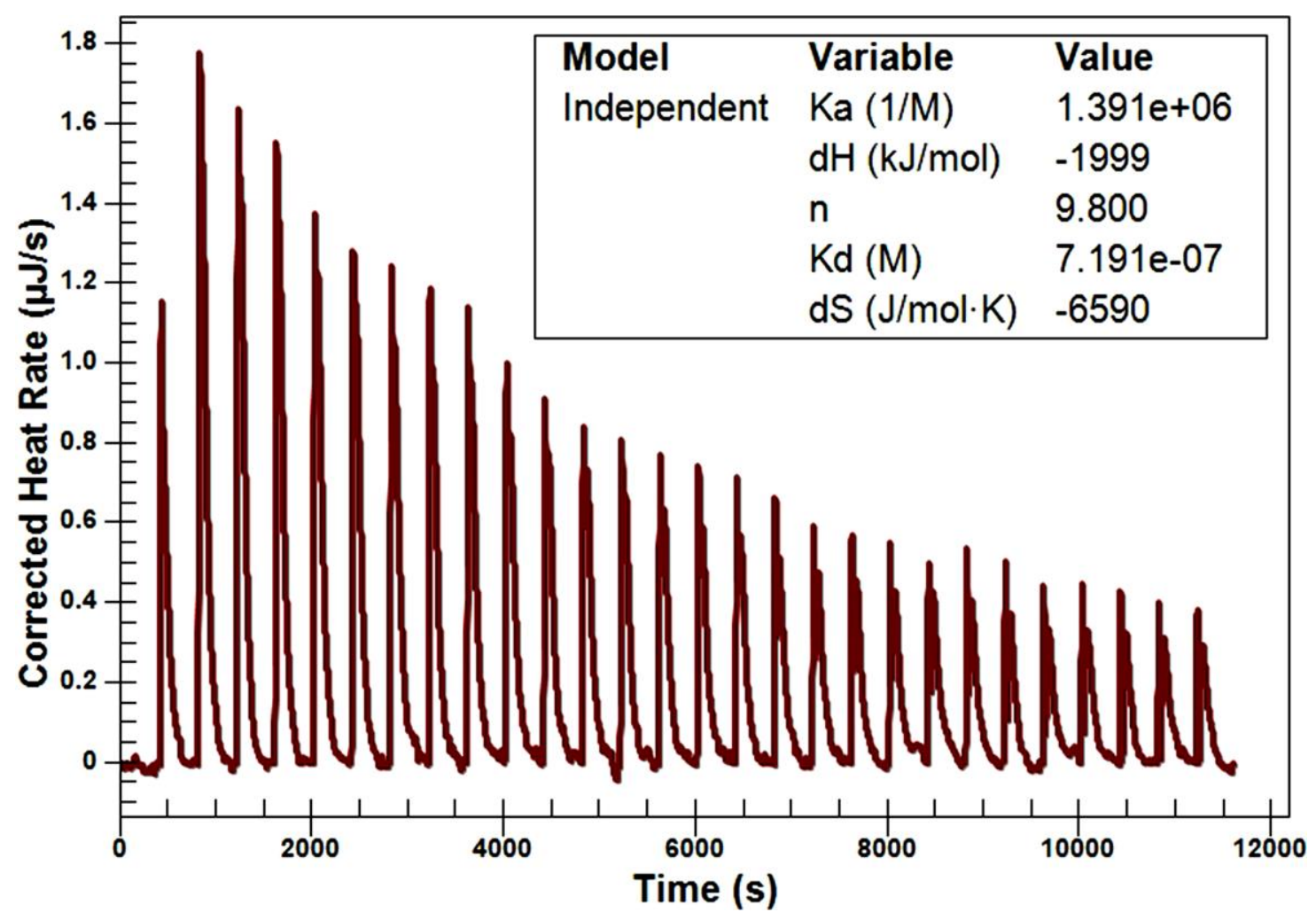

Figure 7: Isothermal calorimetric titration $(\mathrm{T}=298 \mathrm{~K})$ of $\mathrm{MspA}\left(1.5625 \times 10^{-7} \mathrm{M}\right)$ with $\mathrm{RuC2}(0$ to $\left.1.5625 \times 10^{-6} \mathrm{M}\right)$ in $1 \times$ PBS. 
It is noteworthy that binding of $\mathrm{RuC} 2$ to $\mathrm{MspA}$ results in enthalpy-entropy compensation, which is the hallmark of stable supramolecular interactions, as indicated by the binding constant. ${ }^{24,25}$

\section{CONCLUSION}

We have investigated the ability of the mycobacterial porin MspA to act as a model system for the development of channel blockers as novel type of anti-TB drugs. The concept that Ruthenium(II)quaterpyridinium complexes have the capability to act as efficient channel blockers for MspA and related porins has emerged after very high binding constants, $1.1 \times 10^{9}$ $\mathrm{M}^{-1}$ for RuC4 to $7.5 \times 10^{9} \mathrm{M}^{-1}$ for $\mathrm{RuCl}$, have been measured by HPLC and steady-state luminescence. ${ }^{10}$ MspA features a funnel-like structure and a high density of negative charges in its interior, as well as a pronounced constriction zone (bottleneck). Here, we have revisited this approach. Time-resolved absorption measurements indicated that MspA has the ability to participate in photo-induced electron transfer processes from the $* \mathrm{Ru}^{\mathrm{II}}-\mathrm{py}^{\mathrm{I}}{ }^{3} \mathrm{MLCT}$-states of $\mathrm{Ru}(\mathrm{II})$ quarter-pyridinium complexes to their external bipyridinium units. Time-resolved emission studies indicated that the luminescence lifetimes of MspA-bound $\mathrm{Ru}(\mathrm{II})$ quaterpyridinium complexes is shorter than in their free state, which can be explained by the binding of more than one Ru(II)complex to MspA. Atomic Force Microscopy studies of the $\mathrm{Ru}(\mathrm{II})$ quaterpyridinium complex $\mathrm{RuC2}$, MspA and mixtures of $\mathrm{RuC2}$ and MspA on mica have confirmed that RuC2 and MspA do not form 1:1 supramolecular aggregates, but extensive clusters in the 2-dimensional system. Dynamic Light Scattering studies in solution confirmed the findings by $\mathrm{AFM}$ on mica. RuC2 and MspA form large clusters with interesting surface characteristics in PBS. Isothermal Titration Calorimetry has shown that the binding constant of 
$\mathrm{RuC} 2$ to MspA in PBS is $1.4 \pm 0.1 \times 10^{6} \mathrm{M}^{-1}$, and that the ratio of $\mathrm{RuC} 2$ to MspA that permits the best fit of the experimental data is close to 10:1.

All four studies (time resolved absorption/emission, AFM, DLS, and ITC) provide evidence for the formation of clusters/large aggregates of $\mathrm{RuC} 2$ and MspA. Therefore, it has been confirmed that the binding of prospective channel blockers to porins that are not firmly immersed in membranes, cannot be investigated without the occurrence of large errors that arise from the formation of large supramolecular aggregates between porins and channel blockers. This principal complication has to be taken into account when discussing channel blocking experiments.

\section{EXPERIMENTAL}

\section{Dynamic Light Scattering and Zeta Potential Measurements}

Dynamic light scattering and zeta potential measurements were done using ZetaPALS Zeta Potential Analyzer purchased by Brookhaven Instruments Corporation. For all experiments $50 \mu 1$ of WT MspA ( $\sim 0.6 \mathrm{mg} / \mathrm{ml})$ was used in $2.0 \mathrm{ml}$ of 1x PBS. The number of moles of MspA in 50 $\mu \mathrm{l}$ is calculated to be $1.875 \times 10^{-10} \mathrm{~mol}$. Three mixtures of RuC2 and MspA were prepared with varying concentrations of RuC2:MspA that are 10:1, 100:1 and 1000:1 (i.e. $1.875 \times 10^{-9}, 1.875 \mathrm{x}$ $10^{-8}, 1.875 \times 10^{-7} \mathrm{~mol}$ 's of RuC2 per $1.875 \times 10^{-10}$ mol's of MspA in $2.0 \mathrm{ml}$ of $\left.1 \times \mathrm{PBS}\right)$. Average effective diameter and zeta potential measurements of MspA-RuC2 aggregates were recorded with increasing temperature values of $25,30,35,40,45,50,55,60,65$ and $70{ }^{\circ} \mathrm{C}$.

\section{Steady-state absorption and photolysis}

Absorption spectra were recorded using a HP 8543A UV-Vis spectrometer. The solvent was PBS (phosphate buffered saline). 
Laser flash photolysis: Transient absorption spectra and lifetimes were measured on a homebuilt instrument pumped by a frequency tripled $(355 \mathrm{~nm})$ Spectra-Physics GCR-150 Nd: YAG laser (fwhm $8 \mathrm{~ns}, 5 \mathrm{~mJ}$ per pulse). The output from a $150 \mathrm{~W}$ Xe arc lamp (USHIO) powered by a PTI PS-220 power supply was focused onto the sample at $90^{\circ}$ with respect to the laser beam. The white light transmitted by the sample was collimated and focused onto the entrance slit of a Spex HR-20 single monochromator(1200 gr/mm) and was detected utilizing a Hamamatsu R928 photomultiplier tube and processed by a Tektronics $400 \mathrm{MHz}$ oscilloscope (TDS 380). ${ }^{26-28}$

Atomic Force Microscopy (AFM) Measurements: AFM images were recorded using the PicoScan 2000 AFM (Agilent Technologies) in the magnetic ac mode (MACmode). ${ }^{17}$ MacMode type II tips from Agilent Technologies were used (tip radii $<7 \mathrm{~nm}$, nominal $\mathrm{k}$ value ) 2.8 $\mathrm{N} / \mathrm{m}$, resonance frequency ) $50-75 \mathrm{kHz}$ in air). The size of the images was corrected according to the results from a calibration procedure using tris- homoleptic ruthenium(II)-quaterpyridinium complexes as model compounds. ${ }^{8}$

Mica plates from Mica \& Micanite Supplies, Ltd., England, were used as the model adsorbent. The sheets were freshly formed before each experiment and used without further pretreatment. The bare mica surface is negatively charged in aqueous solutions, with a charge density of -2.1 $\mathrm{e} / \mathrm{nm}^{2}{ }^{29}$ The electrokinetic characteristics of the bare mica surface in the supporting electrolyte solution $(\mathrm{NaCl})$ were determined from streaming potential measurements, such as those described by Scales et al. ${ }^{30}$ The zeta potential of the bare mica surface increases monotonically with increasing the ionic strength, reaching $-87 \mathrm{mV}$ at $\mathrm{I}=1 \times 10^{-3} \mathrm{M}$ and $-38 \mathrm{mV}$ at $\mathrm{I}=1 \times 10^{-2} \mathrm{M}$ at $\mathrm{pH}=7.4$. Spincasting was performed at 8000 RPM using a self-made apparatus. The samples 
were incubated at $293 \mathrm{~K}$ for $24 \mathrm{~h}$ under air prior to the AFM measurements. We have investigated the statistical distribution of the pore diameters employing the program image, which is available from the National Institutes of Health (NIH). ${ }^{19}$

\section{Isothermal Titration Calorimetry}

ITC experiments were performed using a commercial calorimeter Nano ITC-Waters LLC at $298 \mathrm{~K}$ with a cell volume of $1.25 \mathrm{~mL}$. A 1 X PBS buffer was used as solvent. The concentrations of the stock solutions were $1.5625 \times 10^{-7} \mathrm{M}(\mathrm{MspA})$ and $1.5625 \times 10^{-6} \mathrm{M}(\mathrm{RuC} 2)$, respectively. Experiments were done in double runs where 30 injections of $8 \mu \mathrm{L}$ of guest solution were added to a solution of host in the cell at intervals of $400 \mathrm{~s}$. The standard NanoAnalyze software was employed for integration of heat changes for each step in the titration (see Figure 7) and in the subsequent modeling. The design of the ITC measurements and the analysis of the data was performed as described in ref 31 .

\section{ASSOCIATED CONTENT}

Supporting Information. AFM images for MspA, RuC2, and RuC2@MspA (stoichiometric ratios 10:1 and 100:1), a description of the analysis of the AFM images using the program IMAGE/NIH, and control experiments for the ITC measurements. This material is available free of charge via the Internet at http://pubs.acs.org.

\section{AUTHOR INFORMATION}

Corresponding Authors 
*Dr. Stefan H. Bossmann, and Dr. Ayomi S. Perera, Kansas State University, Department of

Chemistry, CBC Building 201, Manhattan, KS 66506-0401, USA; sbossman@ksu.edu;

ayomee@ksu.edu, as well as Dr. Claudia Turro, The Ohio State University, Department of

Chemistry and Biochemistry, 100W, 18St., Columbus, OH 43210; turro@chemistry.ohio-

state.edu

\section{Author Contributions}

The manuscript was written through contributions of all authors. All authors have given approval to the final version of the manuscript.

\section{Funding Sources}

This material is based upon work supported by the National Science Foundation under Award No. EPS-0903806 and matching support from the State of Kansas through Kansas Technology Enterprise Corporation.

\section{References}

1. World Health Organization, Global Tuberculosis Control 2011, ISBN 9789241564380.

2. Villemagne, B.; Crauste, C.; Flipo, M.; Baulard, A. R.; Deprez, B.; Willand, N. Tuberculosis: The drug development pipeline at a glance Eur J Med Chem 2012, 51, 1-16.

3. Lamichhane, G. Novel targets in M. tuberculosis: search for new drugs Trends Mol Med 2011, 17, 2533.

4. Niederweis, M.; Bossmann, S. H.; American Scientific Publishers: 2004; Vol. 7, p 851-67

5. Niederweis, M.; Ehrt, S.; Heinz, C.; Klocker, U.; Karosi, S.; Swiderek, K. M.; Riley, L. W.; Benz, R. Cloning of the mspA gene encoding a porin from Mycobacterium smegmatis Mol Microbiol 1999, 33, 933-45.

6. Faller, M.; Niederweis, M.; Schulz, G. E. The Structure of a Mycobacterial Outer-Membrane Channel Science (Washington, DC, U. S.) 2004, 303, 1189. 
7. Leaym, X.; Kraft, S.; Bossmann, S. H. Synthesis of water-soluble highly charged and methylenebridged resorcin[4]arenes Synthesis 2008, 932-42.

8. Shi, A.; Pokhrel, M. R.; Bossmann, S. H. Synthesis of highly charged ruthenium(II)-quaterpyridinium complexes: a bottom-up approach to monodisperse nanostructures Synthesis 2007, 505-14.

9. Pokhrel, M. R.; Gamage, P.; Kalita, M.; Shi, A.; Bossmann, S. H. Developing new strategies for the treatment of tuberculosis employing ruthenium(II)quaterpyridyl complexes J. Nepal Chem. Soc. 2009, 23, 2-10.

10. Dani, R. K.; Kang, M.; Kalita, M.; Smith, P. E.; Bossmann, S. H.; Chikan, V. MspA Porin-Gold Nanoparticle Assemblies: Enhanced Binding through a Controlled Cysteine Mutation Nano Lett. 2008, 8, 1229-36.

11. Basel, M. T.; Dani, R. K.; Kang, M.; Pavlenok, M.; Chikan, V.; Smith, P. E.; Niederweis, M.; Bossmann, S. H. Direct Observation of Gold Nanoparticle Assemblies with the Porin MspA on Mica ACS Nano 2009, 3, 462-6.

12. Mailaender, C.; Reiling, N.; Engelhardt, H.; Bossmann, S.; Ehlers, S.; Niederweis, M. The MspA porin promotes growth and increases antibiotic susceptibility of both Mycobacterium bovis BCG and Mycobacterium tuberculosis., Microbiology 2004, 150, 853-864.

13. Bossmann, S. H.; Turro, C.; Schnabel, C.; Pokhrel, M. R.; Payawan, L. M., Jr.; Baumeister, B.; Woerner, M. Ru(bpy) ${ }_{3}{ }^{2+} / \mathrm{TiO}_{2}$-Codoped Zeolites: Synthesis, Characterization, and the Role of $\mathrm{TiO}_{2}$ in Electron Transfer Photocatalysis J. Phys. Chem. B 2001, 105, 5374-5382.

14. Turro, C.; Bossmann, S. H.; Leroi, G. E.; Barton, J. K.; Turro, N. J. Ligand-Specific Charge Localization in the MLCT Excited State of $\mathrm{Ru}(\text { bpy })_{2}(\text { dpphen })^{2+}$ Monitored by Time-Resolved Resonance Raman Spectroscopy Inorg. Chem. 1994, 33, 1344-1347.

15. Glazier, S.; Barron, J. A.; Houston, P. L.; Abruna, H. D. Photophysics of PAMAM-Based Dendrimers of Polypyridyl Complexes of Ruthenium J. Phys. Chem. B 2002, 106, 9993-10003.

16. Harriman, A. Further comments on the redox potentials of tryptophan and tyrosine J. Phys. Chem. 1987, 91, 6102-6104

17. Han, W.; Lindsay, S. M.; Jing, T. A magnetically driven oscillating probe microscope for operation in liquids Appl. Phys. Lett. 1996, 69, 4111-4113.

18. Jachimska, B.; Kozlowska, A.; Pajor-Swierzy, A. Protonation of Lysozymes and Its Consequences for the Adsorption onto a Mica Surface Langmuir 2012, 28, 11502-11510.

19. www.NIH.gov/IMAGE

20. Perera, A. S.; Wang, H.; Shrestha, T. B.; Troyer, D. L.; Bossmann, S. H. Nanoscopic Surfactant Behavior of the Porin MspA in Aqueous Media Beilstein J. Nanotechnol. 2012, submitted.

21. Braun, J.; Renggli, K.; Razumovitch, J.; Vebert, C.; John Wiley \& Sons Ltd.:Supramolecular Chemistry: From Molecules to Nanomaterials, 2012; Vol. 2, p 411-424.

22. Israelachvilli, J. N.; Mitchell, D. J.; Ninham, B. W. Theory of self-assembly of hydrocarbon amphiphiles into micelles and bilayers J. Chem. Soc., Faraday Trans. 2 1976, 72, 1525.

23. Doane, T. L.; Chuang, C.-H.; Hill, R. J.; Burda, C. Nanoparticle zeta-Potentials Acc. Chem. Res. 2012, $45,317$.

24. Williams, D. H.; Stephens, E.; O'Brien, D. P.; Zhou, M. Understanding noncovalent interactions: ligand binding energy and catalytic efficiency from ligand-induced reductions in motion within receptors and enzymes Angew Chem Int Ed Engl 2004, 43, 6596-6616.

25. Olsson, T. S.; Ladbury, J. E.; Pitt, W. R.; Williams, M. A. Extent of enthalpy-entropy compensation in protein-ligand interactions Protein Sci 2011, 20, 1607-1618. 
26. Shrestha, T. B.; Melin, J.; Liu, Y.; Dolgounitcheva, O.; Zakrzewski, V. G.; Pokhrel, M. R.; Gogritchiani, E.; Ortiz, J. V.; Turro, C.; Bossmann, S. H. New insights in the photochromic spirodihydroindolizine/betaine-system Photochem. Photobiol. Sci. 2008, 7, 1449-1456.

27. P. M. Bradley, B. E. Bursten and C. Turro, Excited-State Properties of $\mathrm{Rh}_{2}\left(\mathrm{O}_{2} \mathrm{CCH}_{3}\right)_{4}(\mathrm{~L})_{2}(\mathrm{~L}=$ $\mathrm{CH}_{3} \mathrm{OH}, \mathrm{THF}, \mathrm{PPh}_{3}$, py), Inorg. Chem., 2001, 40, 1376-1379

28. J. T. Warren, W. Chen, D. H. Johnston and C. Turro, Ground-State Properties and Excited-State Reactivity of 8-Quinolate Complexes of Ruthenium(II), Inorg. Chem, 1999, 38, 6187-6192

29. Rojas, O. Adsorption of polyelectrolyte on mica. In Encyclopedia of Surface and Colloid Science; Marcel Dekker: New York, 2002; p 517.

30. Scales, P. J.; Grieser, F.; Healy, T. W.; White, L. R.; Chan, D. Y. C. Electrokinetics of the silicasolution interface: a flat plate streaming potential study, Langmuir 1992, 8, 965. 